\title{
Student Video-Usage in Introductory Engineering Courses
}

\author{
Negar M. Harandi*, Farshid Agharebparast*, Luis Linares*, Samuel Dodson*, Ido Roll*, \\ Matthew Fong*, Dongwook Yoon* and Sidney Fels* \\ *University of British Columbia \\ negarm@ece.ubc.ca
}

\begin{abstract}
As videos are gaining popularity in flipped and blended Engineering classrooms, there is an increasing need to track and understand students' use of the videos, in order to identify evidence-based practices matched to the emerging trends in video and video annotation tools. We explore students' survey responses, follow-up interviews, and log data from their interaction with common video platforms as well as, ViDeX, a new experimental video annotation tool, to evaluate how, when and why students watch, rewatch, and annotate videos in two large introductory Engineering courses, with flipped, and blended formats. Our findings show that students watch the videos with the instructors' intended use in mind, and plan their review process accordingly. In the flipped classroom, most students summarized the short preclass screencasts in their personal notes to minimize the need to re-watch the videos before the exam. In contrast, students in the blended classroom reexamined the long tutorial videos mostly to redo the problems before the midterm and final exams. Bookmarking seemed to be useful for locating those problems of interest. Since the problems required drawings and computations, paper annotation was more beneficial than a dedicated video annotation platform.
\end{abstract}

Keywords: Learning Technology, Blended Learning, Learning analytics, Educational Videos, Annotation, Introductory Engineering.

\section{INTRODUCTION}

Videos and screencasts [1] are gaining more popularity as educational tools in e-learning [2] and MOOCs [3], as well as in blended and flipped classrooms [4]. Several studies rely on students' selfreports to assess the perceived value of educational videos. In a recent example, Long et al. [5] carried out a survey in an undergraduate science course, concluding that instructor-developed videos are the most desired type of pre-class material in a flipped classroom model. However, not all surveys indicate an equally positive attitude among students to the use of educational videos. One example is the extensive study by Jaster [6] that reveals students in a flipped college algebra classroom indicated a mixed perception of video viewing, with the majority complaining about not being able to ask questions while watching the videos.

Recently, researchers have started to explore usertrace data to validate students' self-reports and make evidence-based recommendations regarding the development and deployment of educational videos. Analytics tools such as SocialSkip [7] have been developed to compute user activity graphs of online videos. In the Performing Arts discipline, the videoannotation software Collaborative Lecture Annotation System has been used to explore types of learning profiles evolving from student use of video content, with one study concluding that more students fall into passive viewing behaviour if no reward (e.g. grades) is associated with the active video interaction [8]. In a flipped Computer Science classroom, a collaborative streaming platform called $\operatorname{Tr} A C E$ was used to measure students' consumption of educational videos [9]. The research found that students often have low compliance with video viewing expectations, and that re-watching course content occurs infrequently. In a small third year Engineering project course, analytics data from Moodle (moodle.org) and YouTube was used to explore students' viewership trends [10]. The study showed that students complied with the course instructions, watching the videos mostly during class time with group mates, while working on a project.

In this paper, we describe students' use of educational engineering videos, as reflected in our mixed-methods approach, synthesizing data from three different types of sources: activity logs, a survey and interviews. In engineering education, this work is the very first to use the data analytics approach to explore students' interaction with instructor-made video materials in large classrooms. Our data comes from two large introductory engineering courses using the flipped and blended classroom style. Students in our study used a novel video annotation tool [10], as well as the university's Learning Management System (LMS), and YouTube. Triangulation of these multiple approaches provides multiple pathways for gaining insights into students' active engagements with video, 
such as if, when, why, and how they watch, re-watch, and annotate video content for their learning.

\section{MATERIALS AND METHODS}

This section describes the materials and procedure of our study.

\subsection{Video Materials}

Educational videos were used as supplementary material in two introductory engineering courses at University of British Columbia (UBC) in the fall of 2017. In a first-year Applied-Science course (APSC), 13 short videos $(7.5 \pm 3.3$ minutes) served as weekly pre-class materials. The course had 460 student enrolments across three sections. On each class day, there were three sections with about 190 students having the class in the morning and 270 students the same class in the afternoon. The APSC videos used for this study were only available in the second-half of the semester. In a second-year Electrical-Engineering course (ELEC), 33 videos with varied length (18.9 \pm 12.9 minutes) served as supplementary material for the assignments and bi-weekly midterm exams. The course had 199 student enrolments across two sections, with half of the students having the class in the morning, and the other half in the afternoon. The ELEC videos were only administered during the firsthalf of the semester; however, students could access the videos throughout the semester for their own review purposes.

Watching the videos was expected by the instructors in each course but remained optional and did not explicitly count for any marks in either course. In both courses, the videos were gradually released, according to the material that was covered, or expected to be covered in the classroom.

\subsection{Video Platforms}

To record student interactions with the videos, we made them available in ViDeX [11], a video annotation tool that is under development at UBC in collaboration with Microsoft. As a free-of-charge, web-based video player, ViDeX provides students with extra affordances to annotate video by highlighting and taking notes within its platform. Figure 1 shows a screenshot of one video lesson in ViDeX, as seen by the students. The interface has four main components: The Video Player, the Filmstrip, the Transcript, and the student's Notes. The Filmstrip supports visual navigation through multiple thumbnails of the video. The Transcript enables students to browse through transcribed text, to read ahead, and to scan or search for keywords in the video without having to explicitly watch it. ViDeX allows students to create their own highlight or notes in each

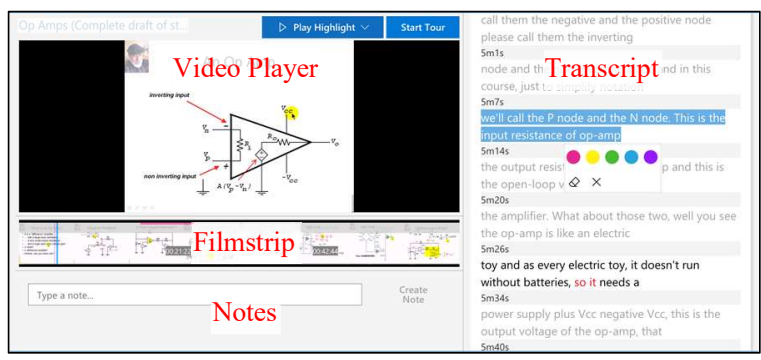

Fig. 1. Screenshot of student view of a video lesson in ViDeX. Students can highlight either on the Transcript, or Filmstrip, and create their timestamped notes.

video lesson. After clicking on and dragging across either the Filmstrip or the Transcript, students can choose a colour using a popup widget to highlight the selected interval (as depicted in Figure 1). The components are synced, so a highlight using the Filmstrip, for example, shows up in the Player and the Transcript. Highlights of a particular colour can be played back selectively using the navigation controls. Students can also write short notes that are attached to specific time stamps in the video.

Prior to using ViDeX for the first time, students were provided with a consent form that allowed the ViDeX team to analyze the data logs; and all student data was recorded, and stored anonymously in accordance with the provincial legislation.

The videos were also made available outside of ViDeX which moreover catered to the needs of the students who wanted to opt out of the ViDeX experiment. APSC videos were shared through the university LMS. Every week new videos were released and advertised on their corresponding LMS folder, bearing the links to both the ViDeX and LMS version of the videos. Students were expected to watch a few videos every week, and were quizzed on the corresponding video content later in class using iClickers (iclicker.com) - a radio-frequency based interactive in-classroom selection device. ELEC videos were all included in a playlist on YouTube, under a channel moderated by the instructor [redacted link]. It is worth noting that this channel has more than $30 \mathrm{~K}$ subscribers, and is well-known to the students. Although most of the videos were from previous years, and consequently readily available on YouTube at the start of the term, some videos were made and added to the playlist as the term progressed. During the semester, the videos (with their YouTube and ViDeX links) were regularly mentioned in form of announcements, and questions and answers posted on the course's discussion forum.

\subsection{Questionnaire and Interviews}

At the end of the semester, we administered a survey to students from both classes. One-on-one 
interviews with students were held to learn more about Engineering students' use of and style of interaction with the course videos.

The online survey was composed of 12 questions. Eight questions were about student perception of, and attitude towards ViDeX as a video player and annotation tool, while other 4 were related to student habits of annotating while watching educational videos. The participants were nominally compensated for their time with a university food services gift card. Forty-eight APSC and 64 ELEC students participated in the online survey. Seventy-three percent of students completed the survey, with the average time being slightly under 5 minutes. It is worth mentioning that the majority of the students surveyed found it easy to find specific content (95\%), to re-watch video content (95\%), to highlight (94\%) and to take notes $(89 \%)$ inside ViDeX.

At the end of the questionaire, students were invited to participate in a short (20 to 30 minutes) one-on-one interview, carried by the Engineering Teaching and Learning Fellow involved in administration of ViDeX videos in their classroom. The interviews followed an unstructured format but touched on several topics including but not limited to student's thought on ViDeX, their use of videos in the course, as well as their annotation habits and needs. Overall, thirteen (10 APSC and 3 ELEC) students participated in the unstructured interviews. The interviews were audio recorded, and later transcribed to text. The participants were nominally compensated for their time with a university food services gift card.

\section{RESULTS AND DISCUSSION}

How often students watched the videos: While the videos were also made available to watch within the university LMS and YouTube, 216 out of 460 (47\%) APSC and 151 out of 199 (76\%) ELEC unique students used ViDeX during the time of the experiment. ViDeX recorded more than $22 \mathrm{~K}$ (APSC) and $69 \mathrm{~K}$ (ELEC) clicks on the video player. Based on the self-reported data in the survey, we divide students into five categories by the number of videos lessons they watched in ViDeX: those who watched $0,1-2,3-$ $5,6-10$, and more than 10 videos. The table below shows the percentage of surveyed APSC and ELEC students in each category.

Table 1. Percentage of surveyed students clustered based on their self-reported ViDeX usage.

\begin{tabular}{|l|l|l|l|l|l|}
\hline \# videos & 0 & $1-2$ & $3-5$ & $6-10$ & $>10$ \\
\hline APSC & $19 \%$ & $19 \%$ & $8 \%$ & $25 \%$ & $\mathbf{2 9 \%}$ \\
\hline ELEC & $6 \%$ & $19 \%$ & $20 \%$ & $\mathbf{3 1 \%}$ & $24 \%$ \\
\hline
\end{tabular}

CEEA18; Paper 080

University of British Columbia; June 3-6, $2018 \quad-3$ of $8-$
Figures 2 and 3 show the number of times each individual video was watched (loaded) in the APSC and ELEC courses respectively, over the time of the experiment. To avoid cluttering the figures, we identify each video with a number tentatively corresponding to their release on the ViDeX platform (i.e., 1 to 13 for APSC and 1 to 33 for ELEC course). Both figures include information about number of times the videos have been accessed in ViDeX, as well as on the LMS (APSC, Figure 2) and on the YouTube Canada (ELEC, Figure 3). As expected, given the familiarity of the LMS and YouTube environments, the number of view counts on LMS and YouTube are larger than ViDeX. In both figures, note that the scale of the right axis is larger than the left, e.g. in Figure 3, video \#29 was watched about 300 times on ViDeX and 600 times on YouTube in Canada. Unfortunately, due to the limitations in YouTube's analytics, we were unable to further separate YouTube's viewing statistics by region or city.

Figures 2 and 3 show some similarity between the watch patterns across different videos on ViDeX, and on LMS or YouTube. As an example, both LMS and YouTube patterns coincide with ViDeX in showing popularity of certain videos over the others. In Figure 2 , video \#2 shows higher watch numbers. This 8minute video introduces a foundational framework and application program interface that is used in the rest of the semester, and hence cited several times in the following videos. Between the ELEC videos in Figure 3, the two most popular were videos \#32 and $\# 29$. Video \#32 is a 64-minute tutorial on a relatively new and complex topic, populated with detailed solution of several exam-like problems. Video \#29 is shorter (31 minutes) and focused more on a familiar but fundamental topic. This video still follows the same tutorial format, as it interweaves the theory with the problem-solving skills.

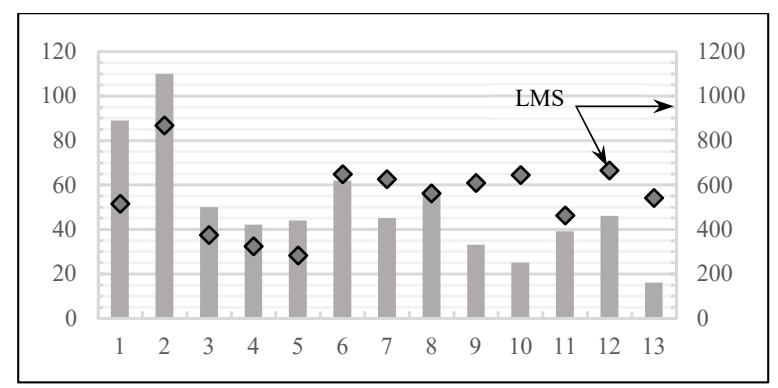

Fig. 2. Aggregate of student views for 13 APSC videos during the experiment in ViDeX (bars, left vertical axis) vs. LMS (diamonds, right vertical axis). Note the 10x difference in the scale of the LMS axis. 


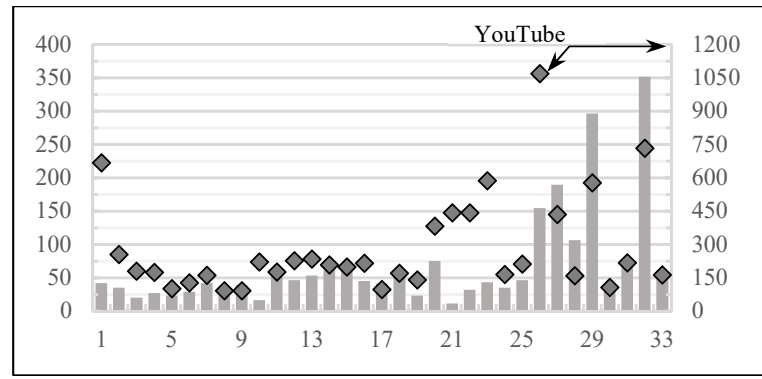

Fig. 3. Aggregate of student views for 33 ELEC videos during the experiment in ViDeX (bars, left vertical axis) vs. YouTube Canada (diamonds, right vertical axis). Note the $4 x$ difference in the scale of YouTube axis.

To make sure that the ViDeX watch patterns were not confounded by information from a few individual users, we also looked at how many times each individual student watched each video. Figures 4 and 5 show the average number of views per student for APSC and ELEC videos respectively. Note that the number of views is normalized by the number of students who watched that particular video. The dashed lines in the figure show the standard deviation. In both cases, the average often falls below two views-

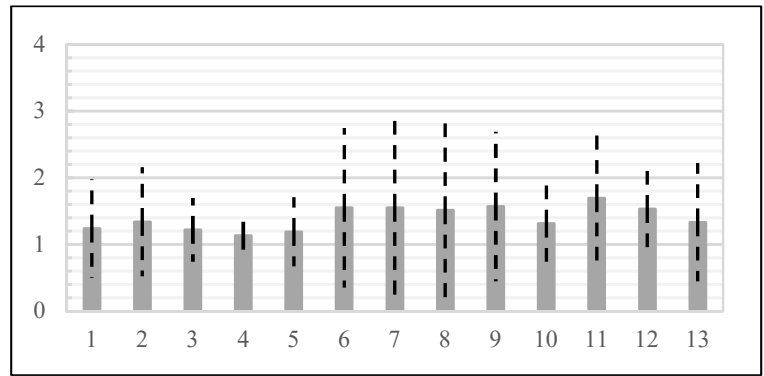

Fig. 4. Average number of ViDeX views-per-student for the 13 APSC videos. dashed lines show the standard deviation.

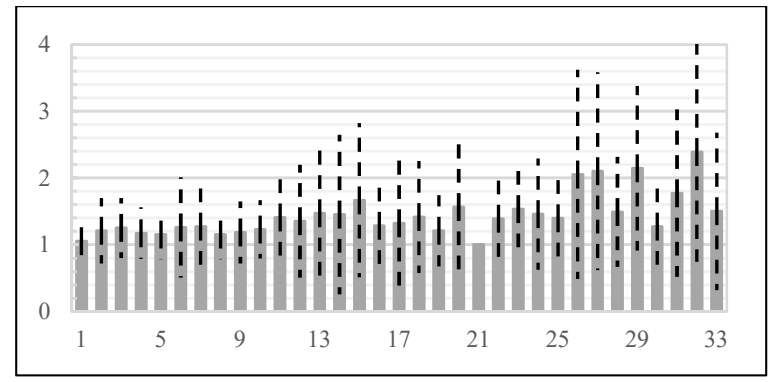

Fig. 5. Average number of ViDeX views-per-student for the 33 ELEC videos. Error bars (dashed lines) show the standard deviation.

per-student, except videos \#32 and \#29, alongside the less popular videos \#26 and \#27, that show higher averages. This data confirms that the most popular videos in both courses were watched more times by more students.

Why and when students watched the videos: Thirty-eight $(80 \%)$ of APSC students who participated in the online survey reported viewing the weekly video lessons before going to the class. In contrast, ELEC students used the videos mainly to prepare for their tests and exams $(55 \%)$ and to review the material after class $(47 \%)$. We observe that these results correlate with the intended use of the videos in each course.

Figure 6 and 7 show the number of views recorded on ViDeX over the experiment time in the APSC and ELEC courses, respectively. Each individual video is coded in a different colour, so that overall, the figures give a sense of the contributions different videos make in the total view counts for each day. The graphs start with the date that ViDeX was introduced to the class and end on the date of final exam. Our log data for each day aggregates the number of views from 12AM to 11:59PM of that day. Note that the $\mathrm{x}$-axis in Figure 7 skips the period between Oct. $9^{\text {th }}$ and Dec. $6^{\text {th }}$, when negligible student activity was recorded on the platform. Both figures also include the total number of views registered outside of ViDeX, i.e. on LMS for the 13 APSC videos and on YouTube Canada for the 33 ELEC videos, over the span of the experiment. Again, while the LMS and YouTube numbers are higher, it is interesting to notice that both datasets show similar patterns in their peaks and valleys.

In order to assess if students were making strategic use of the videos to prepare for course milestones, we looked for correlations between peaks of students' video-usage and the academic calendar of each course. Figure 6 marks the class dates (denoted by CLS) by which students were expected to watch the most recently released videos, on the top, parallel to the date-axis. Figure 7 includes the dates of three (out of five) course midterm exams (denoted by MT\#). The other two midterm exams of the ELEC course were administered in the second half of the term in November, and were related to the material that was not included in the videos. The APSC course did not have any midterm exams in the second half of the semester. Both figures show the date of the final exam (denoted by FNL) as well.

As expected, in the ELEC course (see Figure 7) the dates of the midterm and final exams perfectly correspond with the peaks in the viewing behaviour, suggesting that students used the videos for exam preparation. Though some students started to prepare for their exams as early as the previous weekend, the 


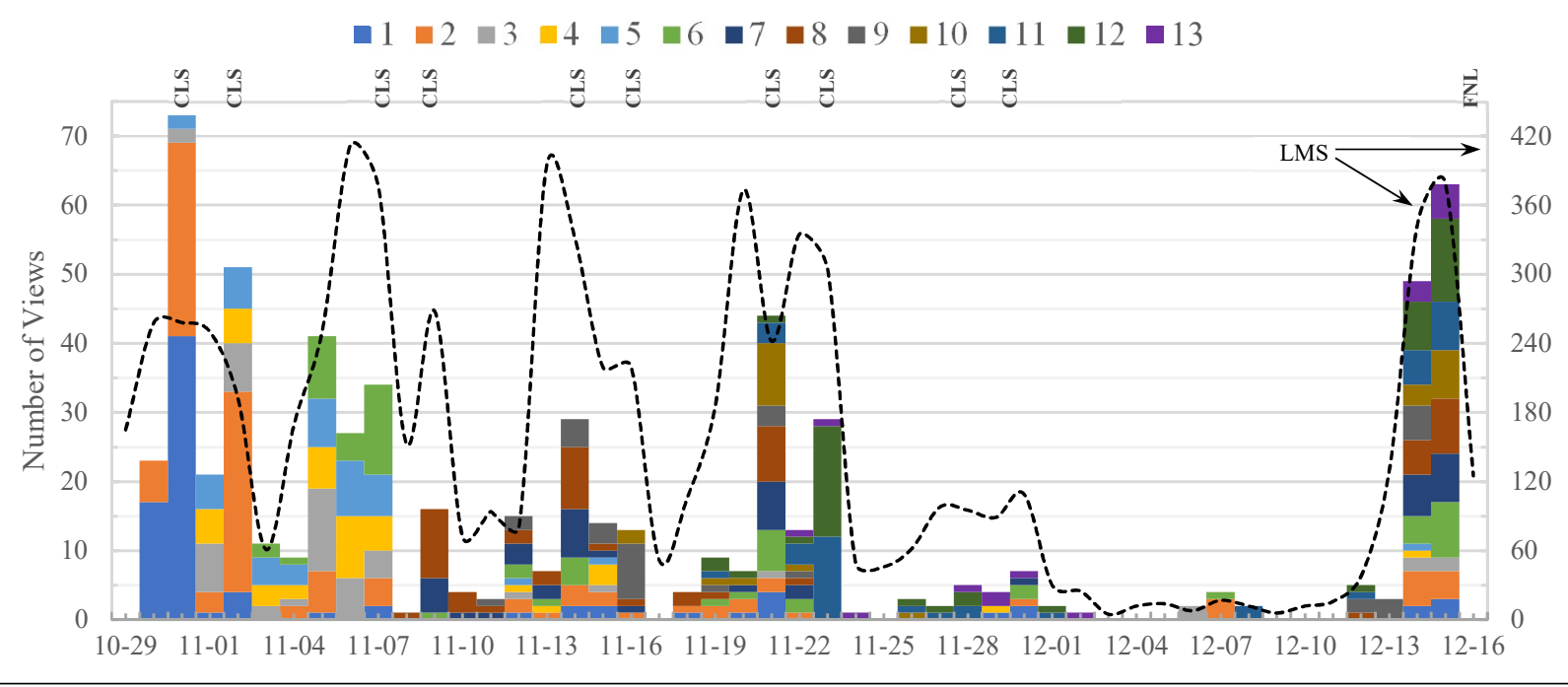

Fig. 6. ViDeX views over time (noted by date) for APSC videos. Each video is identified by a distinct colour. The right vertical axis and the dashed line show the view numbers on the LMS. CLS denotes class dates, FNL denotes the final exam date.

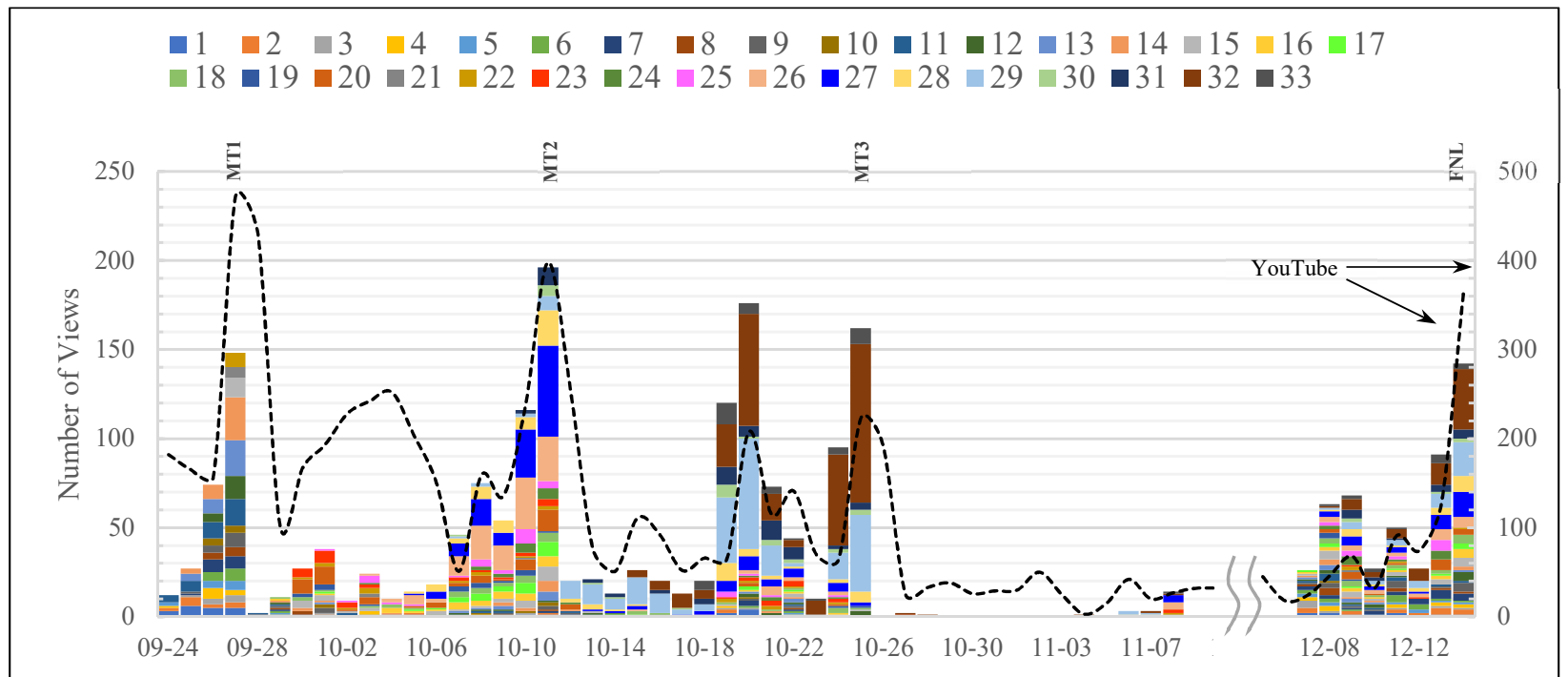

Fig. 7. ViDeX views over time (noted by date) for ELEC videos. Each video is identified by a distinct colour. Note that the $x$-axis excludes the time period between Oct. $9^{\text {th }}$ and Dec. $6^{\text {th }}$ where minimal student activity was registered. The right vertical axis and the dashed line show the view numbers on YouTube Canada. MT\# denotes midterm exam dates, FNL denotes the final exam date.

majority of the views happened the night before, or on the day of the exams, given that midterm and final exams were all scheduled in the afternoon. In the APSC course (see Figure 6), the viewing behaviour shows regular peaks leading to each class, with students watching the videos in a range of about two days up to the day of the class itself. For the final exam, the viewing behaviour was at peak, two and then one day before the exam, but no views were registered on the actual day of the exam, although the exam was scheduled in the afternoon. This is mostly because most students needed to attend another final exam in the morning.

In both figures, the colours in the days before the final exam are denser, but the total view count remains close to the other peaks in each graph. This suggests that students were selective in their review process, only revisiting the videos they deemed important. 
The patterns observed in Figures 6 and 7 corroborate well with what students mentioned in their one-on-one interviews: all 10 APSC interviewees mentioned they watched the videos once (rarely twice) right before each lecture during the term. Five interviewees reported re-watching all or some of the videos one more time before the final exam, while the rest preferred to prepare for the exam using their own notes, or the course handouts. All 3 ELEC interviewees mentioned they watched the videos extensively - usually while they were solving the assignments or studying for the exams, with one of them opting out of spending time on the videos right before the final exam. One student said they watched every single video of the course at least four times, while another mentioned they only watched the videos that were recommended by their colleagues. When comparing Figure 6 and 7, one should remember that more than twice as many students were taking the APSC course than the ELEC course.

Did students annotate? Why and Why not? In response to our survey questions, most of the students (75\% in APSC, 65\% in ELEC) said they do not annotate their textbooks, with the majority $(46 \%$ in APSC, 50\% in ELEC) taking their notes in a separate digital or paper notebook, mainly (40\% in APSC, $38 \%$ in ELEC) to resell their textbooks. When it came to videos, some students (27\% APSC, 17\% ELEC) reported that they used ViDeX affordances to highlight, and create notes in the videos. Overall, only $10 \%$ of the respondents said that they annotated both in the textbook and the ViDeX video lessons. Nineteen percent of students annotated in the text but not in the videos, while $12 \%$ highlighted in the videos but not in the text. The majority, $58 \%$, said they did not annotate either in the text or in the videos.

Using the highlighting feature in ViDeX, 41 APSC students created 314 highlights across the 13 videos. ELEC students showed less interest in video highlighting, resulting in only 14 unique students creating 41 highlights across the 33 videos. In-video notes were less popular than highlights: 24 APSC students created a total of 83 notes on 11 video lessons, with five students accounting for more than $50 \%$ of the notes; numbers were lower for the ELEC course, where 7 students created a total of 12 notes on 6 video lessons. Most students did not create more than one note.

Figures 8 and 9 show the number of highlights, and number of unique students who made those highlights on each video, in the APSC and ELEC course, respectively. Videos \#2 and \#6 in the APSC, and video

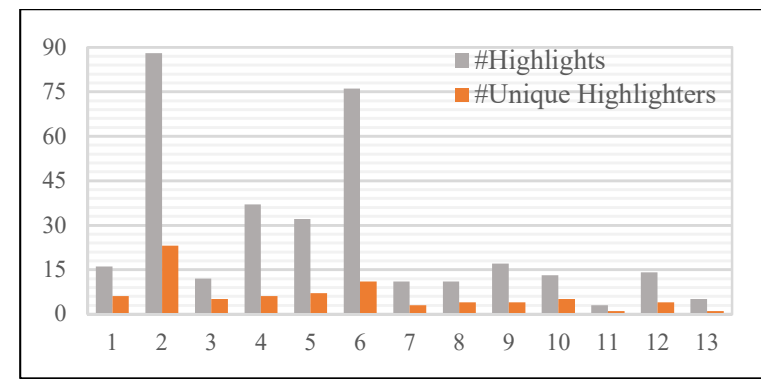

Fig. 8. Number of ViDeX highlights and student highlighters in the 13 APSC videos.

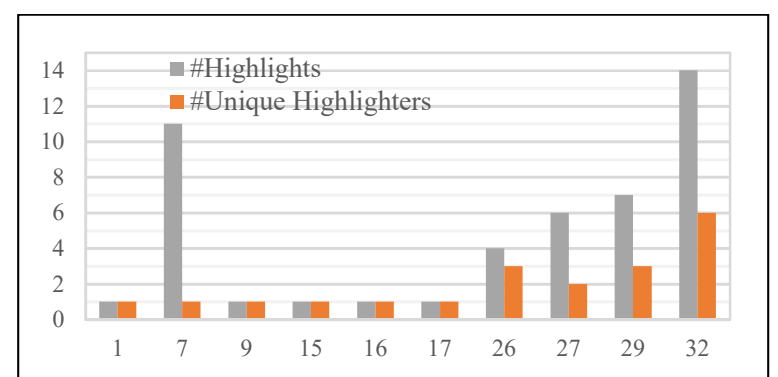

Fig. 9. Number of ViDeX highlights and student highlighters in the ELEC videos. Videos with no student highlight are not included in the figure.

\#32 in ELEC show apparent higher levels of highlights, and highlighters, compared to the others. We looked at the location of the highlights in each of those videos to see whether there is a consensus between learners with regard to valuable video portions. Table 2 shows the heat map for the number of unique highlights over video time for video \#2 and \#6 in the APSC course. The map is normalized by the number of students who highlighted that particular video, so the range varies between 0 and 1 . The video time is shown as a percentage of the video length, with each cell representing $10 \%$ of the video. There is a degree of consensus between position of highlight segments of different students, which suggests that students may be able to benefit from each other's annotations should such crowd-sourcing become available in a future version of ViDeX.

Table 2. Normalized heatmap showing number of student highlights in ViDeX over video time for popular APSC video \#2 (first row) and video \#6 (second row).

\begin{tabular}{|l|l|l|l|l|l|l|l|l|l|}
\hline \multicolumn{8}{|c|}{0} \\
\hline 0.27 & 0.27 & 0.41 & 0.23 & 0.59 & 0.55 & 0.32 & 0.32 & 0.27 & 0.18 \\
\hline 0.64 & 0.27 & 0.55 & 0.18 & 0.27 & 0.45 & 0.27 & 0.36 & 0.18 & 0.27 \\
\hline $0 \%$ & $60 \%$ & $60 \%$ & $80 \%$ & $100 \%$ \\
\hline
\end{tabular}


Apart from the number of the video highlights, we noticed that highlight length also differed between the APSC and ELEC courses. With the exception of a 15minute-long highlight made on video \#32 by one student, most of ELEC highlights were short (median $=3.8$, mean $=5.1$ seconds), probably to serve as bookmarks to certain discussion, problem, or parts of the solutions. APSC highlights were lengthier (median $=6.9$, mean $=12.1$ seconds), probably meant to cover most of the video content that student deemed new or important.

Respondents who reported that they annotated the video lessons in ViDeX were prompted with a followup question that had options they could select specifically about the type and purpose of their annotations. The majority indicated that they highlighted $(76 \%, n=19)$, and created notes $(44 \%$, $n=11$ ), on parts of video that they wanted to re-visit in the future i.e. to help with assignments, tests or exams. Twenty four percent $(n=6)$ indicated they created notes to write down their own thoughts on the material, while $20 \%(n=5)$ indicated they left marks that showed what they have watched so far. In the follow-up interviews, 5 out of 10 APSC students mentioned they used highlighting features in $\mathrm{ViDeX}$ to facilitate relocating of the parts they found important later, for their review before the final exam.

Respondents who reported that they did not annotate the videos in ViDeX were prompted with follow-up options that helped them identify their reasons as well. Several students indicated they did not find highlighting $(45 \%, n=38)$ or note-taking $(26 \%$, $n=22$ ) useful for these specific video lessons (e.g. lessons were straightforward, or short). While others indicated it was distracting to highlight $(42 \%, n=36)$, or create notes $(21 \%, n=18)$ inside ViDeX while they were watching the videos. Adding to these were the others who indicated they did not know that they could highlight $(20 \%, n=17)$ or create notes $(24 \%, n=20)$ using ViDeX. While these covered most of the reasons students did not highlight the videos, note-taking had an additional deterrent. Forty-two percent $(n=35)$ indicated they preferred to record their notes elsewhere (e.g. in a notebook, binder and digital files).

In the follow-up interviews, 4 out of 10 APSC interviewees mentioned they took screenshots of certain frames of the videos and imported them into a digital notebook where they kept their notes made either using a digital pen or by typing. These students preferred having their notes in one place, since they were not planning to watch the videos again. They suggested they would have benefited from potential summarization features that let them export their annotation from the video platform. All three ELEC interviewees mentioned their note-taking routine usually involved drawing circuits and writing equations, which is not currently possible on the ViDeX platform. Two interviewees said they would first watch the lecture parts of the videos, which explained the theory, then pause when the video showed a new problem to do it themselves on pen and pencil. Once they finished solving the problem on their own, the students would resume the video playback to compare their results with what would unfold later in the video.

\section{CONCLUSION AND FUTURE WORK}

Engineering students watched educational videos with different motivations depending on the course they were enrolled in. The frequency of annotating also varied between the two courses.

Students in the flipped APSC classroom were provided with a budgeted number of shorter videos (screencasts) per week. They seem to have used the videos with a shorter-term timeframe in mind, watching the required videos before each session mostly to prepare for the iClicker quizzes at the beginning of each session, and proportionally for the exam preparation. Though videos were released a week in advance, most students watched them closer to (some on the night before, or the day of) their class, usually to stay fresh. Some re-watching of the videos happened before the final exam, nevertheless, interview evidence revealed that the lack of rewatching does not necessarily mean that students have not reviewed the video content, through other mediums, such as their personal notes.

In the lecture-based ELEC course, students used the longer tutorial videos as supplementary material to help with their online graded assignments on the WeBWorK platform. They re-watched the videos at their own pace but extensively before midterm and final exams, mostly to practice the examples again and again.

Annotation habits were idiosyncratic, but showed signs of dependency on the content and intended use of the videos. While ELEC students tended to pause the video, draw and solve the circuits for themselves and resume to check their responses, APSC students took notes inside and outside of the video platform to summarize the videos in their digital or paper notebook for their future review. So compared to APSC students, ELEC students watched more but annotated less videos in ViDeX. In one interesting case, students used highlighting feature of the ViDeX to bookmark a very popular, but long (64 minutes) video tutorial in the ELEC course. 
Though our findings explain some general aspects of student video-usage in the introductory Engineering courses, they are still confined by the contents of the videos, their style, and the role they played in two specific courses. We have only scratched the surface of understanding Engineering students' use of video and video annotation in classes. Thus, we are planning to bring ViDeX to more courses, across several Engineering departments at UBC, to broaden the scope of our findings. In addition, as annotation features in ViDeX evolve, they will become more user-friendly, and hence more able to accommodate study needs of Engineering students. That in turn may result in more consistent use of in situ annotation. As we learn more about video and video annotation behaviours, Engineering instructors can begin to tailor their style and content to best leverage the strengths of video and annotation affordances possible with emerging advances in video viewing technology.

\section{Acknowledgements}

This work is supported in part by a Teaching and Learning Enhancement Fund from the University of British Columbia, the Natural Sciences and Engineering Research Council of Canada, and Microsoft.

\section{References}

[1] Steven A. Lloyd and Chuck L. Robertson, "Screencast tutorials enhance student learning of statistics," Teaching of Psychology, vol. 39, no. 1, pp. 67-71, 2012.

[2] Dongsong Zhang, Lina Zhou, Robert O. Briggs, and Jay F. Nunamaker Jr., "Instructional video in e-learning: Assessing the impact of interactive video on learning effectiveness," Information \& Management, vol. 43, no. 1, pp. 15-27, 2006.

[3] Daniel T. Seaton, Sergiy Nesterko, Tommy Mullaney, Justin Reich, Andrew Ho, and Isaac Chuang, Characterizing video use in the catalogue of MITx MOOCs. Lausanne: European MOOC Stakeholders Summit, 2014, 6 pp.

[4] Jacob Lowell Bishop and Matthew A. Verleger, "The flipped classroom: A survey of the research," in Proc. ASEE National Conf., (Atlanta, GA; 23-26 June 2013), 18 pp., 2013.

[5] Taotao Long, Joanne Logan, and Michael Waugh, "Students' perceptions of the value of using videos as a preclass learning experience in the flipped classroom," TechTrends, vol. 60, no. 3, pp. 245-252, 2016.

[6] Robert Walter Jaster, Inverting the classroom in college algebra: An examination of student perceptions and engagement and their effects on grade outcomes. Texas State University-San Marcos, 2013.

CEEA18; Paper 080

University of British Columbia; June 3-6, $2018 \quad-8$ of 8 -
[7] Michail N. Giannakos, Konstantinos Chorianopoulos, and Nikos Chrisochoides, "Making sense of video analytics: Lessons learned from clickstream interactions, attitudes, and learning outcome in a video-assisted course," The International Review of Research in Open and Distributed Learning, vol. 16, no. 1, 2015.

[8] Negin Mirriahi, Daniyal Liaqat, Shane Dawson, and Dragan Gašević, "Uncovering student learning profiles with a video annotation tool: reflective learning with and without instructional norms." Educational Technology Research and Development, vol. 64, no. 6, pp. 1083-1106, 2016.

[9] Suzanne L. Dazo, Nicholas R. Stepanek, Robert Fulkerson, and Brian Dorn, "An empirical analysis of video viewing behaviors in flipped CS1 courses," ACM Inroads, vol. 7, no. 4. pp. 99-105, 2016.

[10] Laia Albó, Davinia Hernández-Leo, Jaume Barcelo, and Luis Sanabria-Russo, "Video-Based Learning in Higher Education: The Flipped or the Hands-on Classroom?," European Journal of Open, Distance and E-learning, vol. 19, no. 2, 2016.

[11] Samuel Dodson, Ido Roll, Matthew Fong, Dongwook Yoon, Negar M. Harandi, and Sidney Fels. "Active Viewing: A Study of Video Highlighting in the Classroom." in Proc. ACM Conf. on Human Information Interaction \& Retrieval., pp. 237-240. ACM, 2018. 